\title{
BeppoSAX detection and follow-up of GRB 980425
}

\author{
E. Pian $^{1}$, L. Amati $^{1}$, L.A. Antonelli ${ }^{2}$, R.C. Butler ${ }^{3}$, E. Costa $^{4}$, G. Cusumano ${ }^{5}$, J. Danziger ${ }^{6}$, M. Feroci $^{4}$,

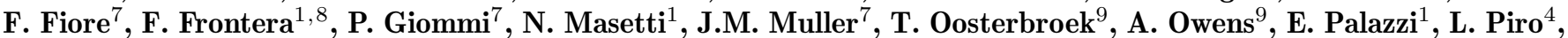 \\ A. Castro-Tirado ${ }^{10}$, A. Coletta ${ }^{7}$, D. Dal Fiume ${ }^{1}$, S. Del Sordo ${ }^{5}$, J. Heise ${ }^{11}$, L. Nicastro ${ }^{5}$, M. Orlandini ${ }^{1}$, A. Parmar $^{9}$, \\ P. Soffitta ${ }^{4}$, V. Torroni ${ }^{7}$, and J.J.M. in 't Zand ${ }^{11}$
}

1 Istituto Te.S.R.E., CNR, via Gobetti 101, I-40129 Bologna, Italy

2 Osservatorio Astronomico di Roma, sede di Monteporzio Catone, Via Frascati 33, 00040 Monteporzio Catone, Italy

3 Agenzia Spaziale Italiana, Viale Regina Margherita 120, Roma, Italy

4 I.A.S., C.N.R., Via Fosso del Cavaliere, Area della Ricerca di Tor Vergata, I-00131 Rome, Italy

${ }^{5}$ I.F.C.A.I., CNR, via Ugo La Malfa 153, I-90146 Palermo, Italy

6 Osservatorio Astronomico di Trieste, Via G.B. Tiepolo 11, I-34131 Trieste, Italy

7 BeppoSAX Scientific Data Center, Via Corcolle 19, I-00131 Rome, Italy

8 Dip. di Fisica, Univ. di Ferrara, Via Paradiso 11, I-44100 Ferrara, Italy

9 Astrophysics Division, SSD of ESA, ESTEC, P.O. Box 299, 2200 AG Noordwijk, The Netherlands

10 IAA-CSIC, Granada, Spain and LAEFF-INTA, Madrid, Spain

11 Space Research Organization Netherlands, Sorbonnelaan 2, 3584 CA Utrecht, The Netherlands

Received March 16; accepted July 5, 1999

\begin{abstract}
We present BeppoSAX GRBM and WFC light curves of GRB 980425 and NFI follow-up data taken in 1998 April, May, and November. The first NFI observation has detected within the $8^{\prime}$ radius error box of the GRB an X-ray source positionally consistent with the supernova SN 1998bw, exploded within a day of GRB 980425, and a fainter X-ray source, not consistent with the position of the supernova. The former source is detected in the following NFI pointings and exhibits a decline of a factor of two in six months. If it is associated with SN 1998bw, this is the first detection of hard X-ray emission from a Type I supernova. The latter source exhibits only marginally significant variability. Based on these data, it is not possible to select either source as a firm candidate for the GRB counterpart.
\end{abstract}

Key words: gamma-rays: bursts

\section{Introduction}

The GRB of 1998 April 25, detected both by the BeppoSAX GRBM and BATSE and localized with arcminute accuracy by the BeppoSAX WFC, stands out for its spatial and temporal coincidence with the optically and radio exceedingly bright Type Ic supernova SN 1998bw (Galama et al. 1998; Kulkarni et al. 1998), in the nearby galaxy ESO $184-$ G82 $(z=0.0085)$. Since the other GRBs for which a redshift measurement is available are located at larger distances $(z \gtrsim 1)$ and are characterized by power-law decaying optical afterglows, in agreement with the "classical" fireball model (Rees \&

Send offprint requests to: pian@tesre.bo.cnr.it
Mészáros 1992), this has raised a debate about a possible association between GRBs and supernovae. Following the detection of GRB 980425, observations of its error box with the BeppoSAX NFI have been activated 10 hours, one week, and six months later. We present here some results and discuss their implications in view of the detection of SN 1998bw in the GRB field. A detailed presentation will be given in Pian et al. (1999).

\section{Data analysis and results}

GRB 980425 triggered the BeppoSAX GRBM at 21:49:11 UT, and was simultaneously detected by the WFC unit 2 (Soffitta et al. 1998). The event had a duration of $31 \mathrm{~s}$ in the range $40-700 \mathrm{keV}$ and of $40 \mathrm{~s}$ in the range $2-26 \mathrm{keV}$, and exhibited a single, non structured peak profile in both bands (Fig. 1). The fluences at $\gamma$ - and hard X-ray energies are $(2.8 \pm 0.5) \quad 10^{-6} \mathrm{erg} \mathrm{cm}^{-2}$ and $(1.8 \pm$ 0.3) $10^{-6} \mathrm{erg} \mathrm{cm}^{-2}$, respectively. (The Galactic absorption in the direction of GRB 980425, $N_{\mathrm{HI}}=410^{20} \mathrm{~cm}^{-2}$, is negligible at energies higher than $2 \mathrm{keV}$.) The BeppoSAX NFI were pointed at the $8^{\prime}$ radius error box determined by the WFC at three epochs starting 10 hours after the GRB (see Table 1; note that the first pointing has been split in two parts). The preliminary analysis of the LECS and MECS data of the first portion of the first pointing shows that inside the WFC error box, two point-like, previously unknown X-ray sources are detected with a positional uncertainty of 1.5: 1SAXJ1935.0-5248 (hereafter S1), at RA $=19 \mathrm{~h} 35 \mathrm{~m} 05.9 \mathrm{~s}$ and Dec $=-52^{\circ} 50^{\prime}$ $03^{\prime \prime}$, and 1SAXJ1935.3-5252 (hereafter S2), at RA $=19 \mathrm{~h}$ $35 \mathrm{~m} 22.9 \mathrm{~s}$ and Dec $=-52^{\circ} 53^{\prime} 49^{\prime \prime}$. Note that the coordinates distributed by Pian et al. (1998) have been revised 
Table 1. Journal of BeppoSAX-MECS observations

\begin{tabular}{cccc}
\hline \hline Date (UT) & $t^{a}(\mathrm{~s})$ & Flux $^{b}\left(\times 10^{-3}\right.$ cts s$\left.^{-1}\right)$ \\
& & $\mathrm{S} 1$ & $\mathrm{~S} 2$ \\
\hline 1998 Apr. 26.334-27.458 & 37220 & $4.6 \pm 0.6^{c}$ & $2.4 \pm 0.5$ \\
Apr. 27.469-28.160 & 21805 & $4.5 \pm 0.7$ & $<2.5$ \\
May 02.605-03.621 & 31975 & $3.0 \pm 0.5$ & $1.4 \pm 0.5$ \\
Nov. 10.754-12.004 & 53122 & $1.8 \pm 0.4$ & $<2.0$ \\
\hline${ }^{a}$ On source exposure time. \\
${ }^{b}$ In the energy range 1.6 - 10 keV. \\
${ }^{c}$ Uncertainties are at 1- $\sigma$; upper limits are at 3- $\sigma$.
\end{tabular}

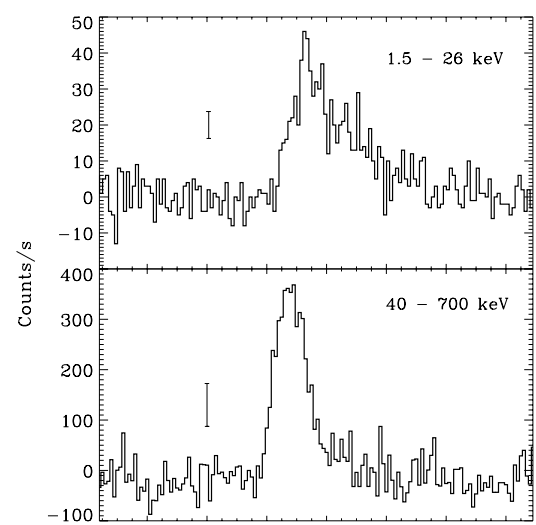

Fig. 1. BeppoSAX WFC (top) and GRBM (bottom) light curves of GRB 980425. The onset of the GRB, indicated by the zero abscissa, corresponds to 1998 April 25.909097 (i.e., 5 seconds earlier than the GRBM trigger time). The vertical bars represent the typical error associated with the individual flux points

in November 1998 (see to this regard Piro et al. 1998). The revised position of $\mathrm{S} 1$ is consistent within the uncertainty with the position of the optical and radio supernova SN 1998bw (Galama et al. 1998; Kulkarni et al. 1998 ), while the revised position of $\mathrm{S} 2$ is $\sim 4^{\prime}$ away from SN 1998bw, and therefore inconsistent with it (see Fig. 1 in Galama et al. 1999). The MECS count rates and upper limits for both sources during the three pointings are reported in Table 1. The upper limits have been estimated by taking into account, besides the normal photon statistics, also the fact that, at these flux levels, the MECS background may be dominated by the fluctuations of the cosmic X-ray background. The observation of November 1998 (taken about a week after the conclusion of this Conference) shows a decrease in the X-ray flux of S1 of approximately a factor of two with respect to the level measured in April-May and the suggestion of slightly extended $\mathrm{X}$-ray emission around the source. During the second portion of the first pointing, as well as in the November pointing, S2 is not detected, while it is detected in the May pointing, at a marginally lower level than in the first observation (see Table 1 ).

\section{Discussion}

The count rates in the first line of Table 1 correspond to $F_{2-10 \mathrm{keV}} \simeq 310^{-13} \mathrm{erg} \mathrm{s}^{-1} \mathrm{~cm}^{-2}$ for $\mathrm{S} 1$ and to
$F_{2-10 \mathrm{keV}} \simeq 1.610^{-13} \mathrm{erg} \mathrm{s}^{-1} \mathrm{~cm}^{-2}$ for S2. The following data points show a decay for $\mathrm{S} 1$ of a factor of two in $\sim 6$ months. Assuming, as suggested by the positional coincidence and by variability, that $\mathrm{S} 1$ is associated with SN 1998bw, the observed variation represents a lower limit on the amplitude of X-ray variability of SN 1998bw. In fact, the possible NFI detection of extended emission indicates that S1 might contain a non negligible contribution from the host galaxy of the supernova. This is the first detection of hard X-ray emission from a Type I supernova. At the distance of SN 1998bw, the luminosity observed in the range $2-10 \mathrm{keV}, 510^{40} \mathrm{erg} \mathrm{s}^{-1}$, is compatible with the luminosity observed in the $0.1-2.4 \mathrm{keV}$ range for the Type Ic SN 1994I, the only case of soft X-ray Type I supernova emission so far detected (Immler et al. 1998). If SN 1998bw is the counterpart of GRB 980425, the production of $\gamma$-rays could be accounted for by the explosion of a very massive star $\left(\sim 40 M_{\odot}\right)$ and by the subsequent expansion of a relativistic shock, in which non thermal electrons are radiating photons of $\sim 100 \mathrm{keV}$, provided the explosion is asymmetric, i.e. the GRB is produced in a relativistic jet (Iwamoto et al. 1998; Woosley et al. 1998; see however, Kulkarni et al. 1998). This raises the hypothesis that two classes of GRBs might exist, with apparently indistinguishable high energy characteristics, but with different progenitors. On the other hand, disregarding the extremely low probability of chance coincidence of GRB 980425 and SN 1998bw, one might consider S2 as the X-ray counterpart candidate of the burst. Assuming a power-law decay between the X-ray flux measured by the WFC in the $2-10 \mathrm{keV}$ range during the GRB and the flux measured in the first NFI observation, we derive a powerlaw index of $\sim-1.4$. The $\mathrm{X}$-ray flux measured in May is however a factor $\sim 10$ larger than implied by the power-law decay. This behavior is unlike that of previously observed X-ray afterglows, although it could be still reconciled with it under the hypothesis of a re-bursting superposed on a "typical" power-law decline.

Acknowledgements. We thank the BeppoSAX Mission Planning Team and the BeppoSAX SDC and SOC personnel for help and support in the accomplishment of this project.

\section{References}

Galama T.J., et al., 1998, Nat 395, 670 Galama T.J., et al., 1999 (this volume) Immler S., et al., 1998, A\&A 336, L1

Iwamoto K., et al., 1998, Nat 395, 672

Kulkarni S.R., et al., 1998, Nat 395, 663

Pian E., et al., 1998, GCN Circular Notice No. 61

Pian E., et al., 1999 (in preparation)

Piro L., et al., 1998, GCN Circular Notice No. 155

Rees M.J., Mészáros P., 1992, MNRAS 258, 41

Soffitta P., et al., 1998, IAU Circ. No. 6884

Woosley S.E., et al., 1998, ApJ (submitted) (astro-ph/9806299) 